Journal of

Epilepsy and

Clinical

Neurophysiology
Patrocinador desta seção:

(1) NOVARTIS

Editor convidado:

ANDRÉ PALMINI

\title{
Dissociação Entre Alterações de Linguagem e Preservação da Musicalidade em uma Criança com Epilepsia Refratária
}

\author{
Denise Ren da Fontoura*, Luciana Tisser**, Júlia Lima do Espírito Santo***, \\ Mirna Wetters Portuguez****, André Palmini***** \\ Serviço de Neurologia, Programa de Cirurgia da Epilepsia, HSL/PUCRS, Porto Alegre, RS
}

\begin{abstract}
RESUMO
Introdução: Estabelecer uma relação entre epilepsia e linguagem é uma tarefa bastante complexa, visto que existem muitas variáveis envolvidas. Dessa forma, podem ocorrer alterações de linguagem expressiva e compreensiva, e em alguns casos a preservação da musicalidade e da prosódia podem estar presentes. Objetivos: $\mathrm{O}$ objetivo deste estudo foi descrever o caso de uma criança com crises epilépticas refratárias multifocais que mostrou uma intrigante dissociação entre o comprometimento de funções de linguagem e a preservação da musicalidade. Métodos: Relato do caso de um menino de 9 anos, internado no Programa de Cirurgia de Epilepsia (PCE) do Hospital São Lucas (HSL) da PUCRS, em novembro de 2004, por apresentar crises epilépticas refratárias. Foram realizados exames clínicos, neurofisiológicos, de neuroimagem e avaliação neuropsicológica. Resultados: Os aspectos lingüísticos do paciente caracterizam uma disfasia, com alterações significativas de expressão e de compreensão da linguagem oral (lobo temporal esquerdo), mas adequação nos aspectos prosódicos da fala e do canto (hemisfério direito). Além disso, a avaliação mostrou um importante atraso global de desenvolvimento neuropsicomotor e alterações comportamentais. Conclusão: Os dados deste trabalho evidenciam a interferência da epilepsia refratária na infância nas habilidades cognitivas, sendo o foco epileptogênico determinante nas alterações lingüísticas. Ressalta-se a importância da avaliação neuropsicológica para mensurar alterações provenientes desta patologia, assim como, identificar áreas da linguagem preservadas, como a musicalidade neste caso, permitindo um elo de ligação para comunicação, avaliação e reabilitação cognitiva.
\end{abstract}

Unitermos: linguagem, epilepsia refratária, alterações de linguagem, avaliação neuropsicológica.

\begin{abstract}
Dissociation between language disorders and musicality preservation in child with refractory epilepsy Introduction: It is a complex task establishing a relation between epilepsy and language since there are so many variables involved. Therefore, expressive and comprehensive language alterations can occur, and in some cases the musical and prosodic preservation can exists. Objectives: The objective of this study was to report the case of a child with multifocal refractory epileptic seizures, and an interesting dissociation between language functions but with musicality preservation. Method: Case report of a 9 year-old child interned in the Epilepsy Surgery Program (PCE) of São Lucas Hospital (HSL) of PUCRS, in November of 2004, presenting refractory epileptic seizures. Clinical, neurophysiologic and imaging exams were done, and also a neuropsychological assessment. Results: The patient's linguistic aspects indicate a dysphasia, with important altercations in oral language's expression and comprehension, but adapted prosodic speech and singing.
\end{abstract}

\footnotetext{
* Fonoaudióloga, especialista em Reabilitação Fonoaudiológica/Voz (IPA) e pós-graduada em Neuropsicologia da Linguagem (HSL/PUCRS). Mestre e doutoranda em Medicina e Ciências da Saúde/Neurociências (FAMED/PUCRS).

** Psicóloga, especialização em Neuropsicologia (ULBRA), pós-graduada em Neuropsicologia (HSL/PUCRS). Mestranda em Medicina e Ciências da Saúde/Neurociências (FAMED/PUCRS).

*** Psicóloga, pós-graduada em Neuropsicologia (ênfase em Epilepsia) e Neuropsicologia Infantil (HSL/PUCRS). Mestranda em Medicina e Ciências da Saúde/Neurociências (FAMED/PUCRS).

**** Coordenadora da Unidade de Neuropsicologia do Serviço de Neurologia do HSL/PUCRS, doutora em Neurociência pela UNIFESP.

***** Diretor Científico, Programa de Cirurgia da Epilepsia, HSL/PUCRS.

Received May 30, 2005; accepted July 22, 2005.
} 
Besides, an important neuropsychomotor development global deficit and behavioral altercations were shown by the assessment. Conclusion: This study's data indicates the refractory epilepsy in childhood interference in cognitive abilities, as the epileptogenic focus is a determinant factor in language disorders. It's remarkable the importance of a neuropsychological assessment to evaluate this pathology's altercations.

Key words: language, refractory epilepsy, language disorders, neuropsychological assessment.

\section{INTRODUÇÃO}

Estabelecer uma relação entre epilepsia e linguagem é uma tarefa bastante complexa, visto que existem muitas variáveis envolvidas, podendo os circuitos neuroanatômicos das áreas de linguagem se sobrepor ao foco epileptogênico ${ }^{(12,14)}$.

Alterações de linguagem no período interictal são freqüentes em crianças com epilepsia, o que sugere dificuldades de comunicação persistentes e não apenas transitórias, durante e após as crises. As relações entre epilepsia e alterações de linguagem passam pela anatomia funcional das áreas cerebrais da linguagem, o que direciona a discussão para pacientes com epilepsias temporais e frontais. Embora espera-se encontrar alterações de linguagem muito mais freqüentemente em pacientes com focos epileptógenos no hemisfério dominante ${ }^{(14)}$. Alguns autores têm mostrado que tanto a epilepsia do lobo temporal direito quanto do esquerdo pode acarretar prejuízos lingüísticos ${ }^{(2,3,15)}$. Além disso, uma relação entre foco epileptogênico, idade de início das crises e funções de linguagem tem sido demonstrada ${ }^{(12)}$, e indivíduos com epilepsias graves têm maior risco de apresentarem alterações de linguagem ${ }^{(14)}$.

A linguagem é uma habilidade complexa e multifacetada que caracteriza-se pela formação de sons, pelo desenvolvimento de sofisticados sistemas de regras e pela existência de uma vasta quantidade de informação, de sentidos e significados ${ }^{(13)}$. Além disto, a linguagem também é expressa por meio de gestos e tom de voz, sendo dependente de aspectos emocionais e tendo importantes componentes afetivos manifestados através da prosódia ${ }^{(5,6,15)}$.

Pacientes com epilepsia podem ter alterações relacionadas às funções "clássicas" da linguagem, como compreensão, expressão, repetição e nomeação ${ }^{(9,14)}$. Freqüentemente estes pacientes apresentam lesões estruturais, incluindo malformações macro ou microscópicas, em estruturas ou circuitos responsáveis pela organização da linguagem no hemisfério dominante. Dependendo do tipo de lesão e da idade do paciente quando a lesão estabeleceu-se ou as crises iniciaram-se, estas funções clássicas da linguagem podem estar representadas de forma mais ou menos exclusiva no hemisfério dominante ou ter havido algum tipo de reorganização funcional, com representa- ção bilateral ou mesmo transferência mais significativa da linguagem para o hemisfério contralateral ${ }^{(4,12)}$.

Entretanto, um outro aspecto bastante intrigante e que começa a ser melhor entendido é a organização diferenciada da linguagem que envolve elementos 'musicais', ou seja, o fato de o cérebro organizar de forma distinta as funções clássicas da linguagem e a 'linguagem musical' musicada). Evidências disponíveis até o momento sugerem que a linguagem associada à musicalidade depende, fundamentalmente, de estruturas localizadas no hemisfério não dominante, o que explicaria a preservação destas funções em pacientes com disfasias clássicas decorrentes de lesões no hemisfério dominante ${ }^{(1,6,7,8,11)}$.

No universo da epileptologia, onde as disfunções e eventuais transferências das funções da linguagem assumem papéis muito relevantes, em especial em candidatos à cirurgia da epilepsia, é crucial identificar-se as relações anátomo-funcionais ligadas às alterações de linguagem clássica e de linguagem musicada.

O estudo descreve uma criança com crises epilépticas refratárias multifocais que mostrou uma intrigante dissociação entre o comprometimento de funções de linguagem mas com preservação da musicalidade.

\section{RELATO DO CASO E MÉTODOS DE AVALIAÇÃO}

I.C. é um menino de 9 anos que internou no Programa de Cirurgia de Epilepsia (PCE) do Hospital São Lucas (HSL) da PUCRS em novembro de 2004, por apresentar crises epilépticas refratárias.

Aos três meses de idade, iniciou com crises tônicoclônicas generalizadas. Com um ano de idade, em decorrência de uma pneumonia, ficou em estado de coma induzido por quatro dias. Freqüentou APAE dos cinco aos seis anos de idade, não sendo encaminhado a nenhuma outra instituição ou escola após esse período.

O desenvolvimento psicomotor de I.C. foi normal, não apresentando alterações até o momento em que foi identificada uma dificuldade na aquisição de linguagem e de controle esfincteriano, que foi realizado aos cinco anos. Quanto ao comportamento apresentava-se hiperativo, com dificuldades na manutenção da atenção, sintomas estes que seguem até o momento.Além disto, não consegue executar tarefas do dia a dia e hábitos de higiene de 
maneira autônoma, tendo que ser orientado e acompanhado em tudo que faz. Segundo relato da mãe, não reconhece perigo, tendo o hábito de fugir quando vê alguma porta ou portão aberto.

Internado no PCE do HSL da PUCRS devido à refratariedade das crises epilépticas, tônico-clônicas generalizadas, com freqüência de três a quatro por semana, a despeito do tratamento com Oxcarbamazepina $1800 \mathrm{mg} /$ dia e Nitrazepam $15 \mathrm{mg} /$ dia. Submeteu-se a exames clínicos, neurofisiológicos, de neuroimagem e avaliação neuropsicológica. Em função das dificuldades do menino, não foi possível obter um resultado quantitativo na avaliação neuropsicológica. Assim, foi realizada uma investigação qualitativa, com enfoque nos aspectos comportamentais e de linguagem. Da mesma forma, em decorrência de suas dificuldades de compreensão, expressão, atenção, concentração e raciocínio, não foi possível aplicar a Escala Wechsler de Inteligência para Crianças (WISC-III), e nem a Escala Colúmbia de Maturidade Mental, tendo em vista sua incapacidade de estabelecer comparações.

Foi utilizada a avaliação qualitativa de Gesell para a determinação da idade mental. Esta avaliação utiliza tarefas padronizadas esperadas para cada faixa etária, servindo de parâmetro para definir uma idade mental. Na avaliação da linguagem, foram investigados aspectos da compreensão e da expressão da linguagem oral. Em relação à compreensão da linguagem oral, foram avaliadas as habilidades de compreensão de ordens simples e complexas, como por exemplo pedir para pegar objetos e mostrar figuras. Quanto à expressão da linguagem oral, avaliou-se a capacidade de comunicação global, de nomeação e de repetição de palavras. Além disto, foram testadas habilidades relacionadas à compreensão e expressão de musicalidade, envolvendo o canto de músicas conhecidas, e perguntas realizadas com exagero da entonação melódica Para adequada avaliação posterior, os testes de linguagem clássica e musical foram gravados em video-tape.

\section{RESULTADOS}

A avaliação neurofisiológica demonstrou desorganização e lentificação dos ritmos de base, com predomínio à esquerda. Além disso, descargas epileptiformes do tipo pontas e pontas-onda, foram registradas de forma multifocal, ocorrendo independentemente, ora à direita, ora à esquerda. Não havia uma localização predominante para estas descargas, embora predominassem nos quadrantes anteriores.

Não foram evidenciadas lesões estruturais à ressonância nuclear magnética de encéfalo.

Durante a avaliação neuropsicológica, mostrou ser uma criança bastante inquieta e impulsiva, não conseguin- do fixar sua atenção por muito tempo em uma atividade, demonstrando dificuldade de inibição dos estímulos. A avaliação da idade mental foi de aproximadamente dois anos e seis meses. $\mathrm{O}$ paciente não compreendia algumas ordens simples como, por exemplo, quando solicitado a mostrar uma figura conhecida, mas compreendia outras, relacionadas às ações do cotidiano. Mostrava também dificuldades para nomear figuras e objetos, mas repetia palavras simples e conhecidas. Não compreendia ordens complexas, mas sua compreensão melhorava levemente quando a pergunta era feita com uma entonação exagerada.

Expressava-se apenas por gestos e vocalizações de vogais, tendo dificuldade em produzir as consoantes em fala coloquial. Apesar disto, apresentava estereotipia caracterizada pela perseveração de algumas palavras ou expressões como "muito bem!", neste caso, com adequada articulação. Interessantemente, entretanto, conseguia cantar músicas conhecidas, apenas com alguns erros articulatórios, e com melodia perfeita. O que evidenciou uma maior facilidade na compreensão e expressão lingüística, quando utilizava-se como método de avaliação, intervenções melódicas, como por exemplo, ao pedir para mostrar um animal, esta pergunta era feita de duas formas, uma com uma entonação usual e outra com entonação exagerada (excessiva modulação de freqüência da fala). Em momentos onde explorava-se potenciais para expressão de linguagem, I.C. continuava a cantar músicas que eram iniciadas por sua mãe, com ritmo e articulação adequados.

\section{DISCUSSÃO}

Os aspectos lingüísticos do paciente caracterizam uma disfasia, com alterações significativas de expressão e de compreensão da linguagem oral, mas adequação nos aspectos prosódicos da fala e do canto. Com tais evidências, acredita-se na funcionalidade de seu hemisfério direito, visto que este atua em certas habilidades artísticas, como a música ${ }^{(7,8,11)}$.

As características referentes à compreensão da linguagem oral evidenciam uma provável disfunção em hemisfério esquerdo, considerado dominante para a linguagem e responsável pela compreensão dos sentidos das palavras (semântica). O exame eletroencefalográfico de I.C. vai de encontro a estes achados, pois mostrou uma assimetria interhemisférica com maior lentificação à esquerda, além de descargas epileptiformes multifocal. Sabe-se que o hemisfério direito também participa do processo de compreensão de palavras, sendo responsável pela prosódia e contribuindo para a compreensão do contexto da mensagem (pré-suposições presentes no tom da voz), completando o processo da fala e da linguagem do hemisfério esquerdo por meio de habilidades de comunicação mais sutis, mas definitivamente importantes ${ }^{(1,6)}$. 
A entonação melódica também está relacionada à aprendizagem lingüística das crianças, facilitando a compreensão e a evocação de uma palavra, reduzindo, assim, as demandas do processo de decodificação ${ }^{(10,11)}$. Em situações com ausência de vocabulário, ocorrem análises de tom e melodia através do hemisfério direito, sendo este capaz de determinar e deduzir fatores emocionais e o contexto do que está sendo dito ${ }^{(6,15)}$. Provavelmente por esse motivo, o paciente obtinha uma melhor compreensão e expressão de ordens simples quando elas eram realizadas com entonação exagerada.

Ressalta-se que, assim como a função de linguagem não está totalmente restrita ao hemisfério esquerdo, o mesmo ocorre em relação à música quanto ao hemisfério direito. Portanto, pode-se dizer que a música, apesar de sua expressão melódica ser elaborada no lobo temporal direito, envolve atividades e funções cerebrais bastante diversas, incluindo o hemisfério esquerdo e sendo caracterizada como uma atividade neuropsicológica comple$\mathrm{xa}^{(6,10,11)}$.

\section{CONCLUSÃO}

A avaliação neuropsicológica qualitativa, enfocando aspectos de linguagem e comportamento, detectou uma disfasia caracterizada por alterações de compreensão e de expressão da linguagem oral, além de um atraso global de desenvolvimento neuropsicomotor e alterações comportamentais.

Os dados desse trabalho evidenciam a interferência da epilepsia refratária na infância, nas habilidades cognitivas, sendo o foco epileptogênico determinante nas alterações lingüísticas. Salienta-se a importância da preservação da musicalidade neste caso, servindo de elo de ligação entre a comunicação, avaliação e reabilitação cognitiva.

\section{REFERÊNCIAS}

1. Baum SR, Dwivedi VD. Sensitivity to prosodic structure in leftand right-hemisphere-damaged individuals. Brain and Language 2003; 87:278-89.

2. Chiron C, Jambaque I, Nabbout R, Lounes R, Syrota A, Dulac O. The right brain hemisphere is dominant in human infants. Brain 1997; 120:1057-65.

3. Hebert S, Racette A, Gagnon L, Peretz I. Revisiting the dissociation betwen singing and a speaking in expressive aphasia. Brain 2003; 126:1838-50.

4. Janszky J, Jokeit H, Heinemann D, Schulz R, Woermann FG, Ebner A. Epileptic activity influences the speech organization in medial temporal lobe epilepsy. Brain 2003; 126: 2043-51.

5. Kandel ER, Schwartz JH, Jessel TM. Fundamentos da neurociência e do comportamento. Rio de Janeiro: Guanabara; 1997.

6. Kotz AS, Meyer M, Alter K, Besson M, Cramon DY, Friederici AD. On the lateralization of emotional prosody: An event-related functional MR investigation. Brain and Language 2003; 86:366-76.

7. McChesney-Atkins S, Davies KG, Montouris GD, Silver JT, Menkes DL. Amusia after right frontal resection for epilepsy with singing seizures: case report and review of the leterature. Epilepsy \& Behavior 2003; 4:343-7.

8. Nicholson KG, Baum S, Kilgour A, Koh CK, Munhall KG, Cuddy LL. Impaired processing of prosodic and musical patterns after right hemisphere damage. Brain and Cognition 2003; 52:382-9.

9. N'Kaoua B, Lespinet V, Barsse A, Rougier A, claverie B. exploration of hemispheric specialization and lexico-semantic processing in unilateral temporal lobe epilepsywith verbal fuency tasks. Neuropsycologia 2001; 39:635-42.

10. Nocolay-pirmolin M. Auditory perception in the child. Acta Otorhinolaryngol Belg 2003; 57:237-41.

11. Peretz I. Brain specialization for Music. The neuroscientist 2002; 8:372-80.

12. Risse GL, Gates JR, Fangman MC. A reconsideration of bilateral representation based on the intracarotid amobarbital procedure. Brain Cogn 1997; 33:118-32.

13. Spring JA, Binder JR, Hammeke TA, Swanson SJ, Frost JÁ, Bellgowan PSF, et al. Language dominance in neurologically normal and epilepsy subjects: a funcional MRI study. Brain 1999; 122:2033-45.

14. Wheless JW, Simos PG, Butler IJ. Language dysfunction in epileptic conditions. Semin Pediatr Neurol 9: 218-28; 2002.

15. Zaidel E, Kasher A, Soroker N, Batori G. Effects of right and left hemisphere damage on performance of the "Right hemisphere communication battery”. Brain and Language 80: 510-535, 2002.

\begin{tabular}{|c|}
\hline Mande seu caso para: \\
JECN - A/c. de ANDRÉ PALMINI \\
Av. Montenegro, 186 sala 505 - Petrópolis \\
CEP 90460-160, Porto Alegre, RS, Brasil ou \\
pelo e-mail: jecnpoa@terra.com.br
\end{tabular}

